\title{
Методика
}

(c) Коллектив авторов, 2020

УДК 612.63.02, 616-092.4

Яковлева А.А.', Павлова Н.Г. ${ }^{2}$

\section{Экспериментальные модели сократительной активности миометрия}

1 ФГБОУ ВО «Первый Санкт-Петербургский государственный медицинский университет имени академика И.П. Павлова» Минздрава России, 197022, г. Санкт-Петербург, Россия, ул. Льва Толстого, д. 6-8, корпус 1;

${ }^{2}$ ФГБОУ ВО «Первый Санкт-Петербургский государственный медицинский университет имени академика И.П. Павлова» Минздрава России, 197022, г. Санкт-Петербург, Россия, ул. Льва Толстого, д. 6-8, корпус 4

Сократительная деятельность матки до настоящего времени остается актуальным вопросом фундаментальных исследований, поскольку отсутствуют единые представления о биомеханике маточного сокращения, необходимые для профилактики родового и акушерского травматизма. Цель работы - оценка ограничений и возможностей экспериментальных моделей, предназначенных для изучения сократительной активности миометрия. Методика. Основными экспериментальными подходами к изучению сократительной активности матки являлись исследования in vitro, in situ, in vivo, in silico, а также их сочетание. Результаты. В статье рассмотрены исследования, в которых использованы различные сочетания экспериментальных подходов, обсуждаются результаты, полученные при моделировании в экспериментах, обсуждаются результаты изучения синхронизации сокращения отделов матки на различных моделях, а также результаты исследования пейсмейкерной активности миометрия и возможность экстраполяции полученных данных на человека. В связи с активным развитием компьютерных технологий в статье поднимается вопрос об их использовании в моделировании сократительной активности матки человека. Заключение. Делается заключение, что комплексный подход, включающий электромиографические, биохимические и морфологические исследования в хроническом эксперименте, является наиболее адекватным для изучения сократительной активности миометрия и функционального состояния нормально развитых и отставших в развитии плодов, что позволит разработать комплексные методы профилактики родового и акушерского травматизма.

Ключевые слова: электромиография; сократительная активность миометрия; экспериментальные модели; функциональное состояние плода.

Для цитирования: Яковлева А.А., Павлова Н.Г. Экспериментальные модели сократительной активности миометрия. Патологическая физиология и экспериментальная терапия. 2020; 64(1): 155-161.

DOI: 10.25557/0031-2991.2020.01.155-161

Для корреспонденции: Яковлева Анастасия Александровна, e-mail: barabulka875@gmail.com

Финансирование. Исследование не имело спонсорской поддержки.

Конфликт интересов. Авторы заявляют об отсутствии конфликта интересов.

Поступила 04.02.2019

Принята к печати 16.01.2020

Опубликована 25.02.2020

Yakovleva A.A. ${ }^{1}$, Pavlova N.G. ${ }^{2}$

\section{Experimental models of myometrium contractile activity}

1 Pathophysiology Laboratory of Pavlov First Saint Petersburg State Medical University,

Russia, St. Petersburg, Lev Tolstoy street 6-8;

${ }^{2}$ Obstetrics, gynecology and reproductology department of Pavlov First Saint Petersburg State Medical University,

Russia, St. Petersburg, Lev Tolstoy street 6-8

Uterine contractile activity remains an important issue of fundamental research as there is no single view of the biomechanics of uterine contraction necessary for the prevention of parturition and obstetric trauma until now. The aim of the review was to assess advantages and limitations of experimental models described in the literature for study uterine contractile activity. At the present time main experimental approaches for study myometrium contractile activity are research in vitro, in situ, in vivo, in silico and the their combinations. The literature presents experimental approaches, different models of uterine contractions synchronization and study of myometrium pacemaker activity. Due to active development of computer technologies there is a 
DOI: 10.25557/0031-2991.2020.01.155-161

need to model human uterine contractile activity with a simplified anatomy. The authors propose that combination of electromyographic, biochemical and morphological methods in chronic experiment is the most correct and appropriate direction for the assessment of the myometrium contractile activity and functional state of normally developed and growth restricted fetuses.

Keywords: electromyography; uterine contractile activity; experimental model; functional fetal state.

For citation: Yakovleva A.A., Pavlova N.G. Experimental models of myometrium contractile activity. Patologicheskaya Fiziologiya $i$ Eksperimental 'naya terapiya. (Pathological Physiology and Experimental Therapy, Russian Journal). 2020; 64(1): 155-161. (in Russian).

DOI: 10.25557/0031-2991.2020.01.155-161

For correspondence: Yakovleva A.A., email: barabulka875@gmail.com

Information about authors:

Yakovleva A.A., https://orcid.org/0000-0002-1889-5928

Pavlova N.G., https://orcid.org/0000-0002-2886-4578

Conflict of interest. The authors declare no conflict of interest.

Acknowledgments. The study had no sponsorship.

Received 04.02.2019

Accepted 16.01.2020

Published 25.02.2020

Сократительная деятельность матки до настоящего времени остается актуальным вопросом фундаментальных исследований, поскольку отсутствуют единые представления о биомеханике маточного сокращения. Понимание механизмов сокращения матки и обусловленного им функционального состояния плода с позиций функциональной системы мать-плацента-плод позволит разработать комплексные методы профилактики родового и акушерского травматизма $[1,2]$.

Известно, что при физиологическом течении родового акта на высоте схватки $\mathrm{PaO}_{2}$ в крови плода снижается всего на 4 мм рт. ст. Следовательно, в таких условиях плод не испытывает гипоксии, поскольку его организм перфузируется запасом оксигенированной крови из венозного синуса [1, 2]. Однако при гипертонической дисфункции или дискоординированной родовой деятельности может наблюдаться гипоксия плода, обусловленная увеличением амплитуды, продолжительности сокращения (систолы) и/или укорочения периода расслабления (диастолы) маточных сокращений [2-4].

В настоящее время основными экспериментальными подходами к изучению сократительной активности матки являются исследования in vitro, in situ, in vivo, in silico и их сочетания.

Moдели in vitro. Большинство исследований in vitro авторы проводят на полосках миометрия, полученных у женщин во время операции кесарева сечения или у экспериментальных животных в конце срока беременности. В литературе приводятся экспериментальные модели in vitro сходного дизайна. Исследование проводят на полосках миометрия, содержащих достаточное количество функциональных сократительных модулей, имеющих прямую механическую связь между собой, при отсутствии в них участков некробиоза. Исследователи погружают полоски миометрия в различные модификации раствора Рингера, а регистрацию миографической активности осушествляют с помощью изометрических и/или изотонических датчиков [5]. В условиях подобной модели авторы изучают экспрессию рецепторов, а также влияние таргетных препаратов на сократительную активность миометрия.

Так в исследовании М. Balki [6] и соавторов удалось проследить восстановление сократительной активности миометрия у женщин после длительной десенсибилизации окситоциновых рецепторов. Авторы показали, что применение больших доз окситоцина (10-15 МЕ) приводит к пролонгированной рефрактерности окситоциновых рецепторов миометрия, что обуславливает длительный более чем 1,5 часовой период восстановления его сократительной активности [6].

На аналогичной модели изучался механизм синхронизации сокращения миометрия в родах [5]. Авторы доказали функциональную и морфологическую однородность миометрия от дна матки до нижнего сегмента. С этой целью иссекался миометрий из разных отделов матки, его равнозначно растягивали, анализируя спонтанную фазовую сократительную активность, реакции на импульсы растяжения и дорастяжения, а также на электрические и химические стимулы. На основании анализа сокращений неодинаково растянутых горизонтальной и вертикальной частей полоски миометрия авторами было установлено, что синхронизация сокращений миометрия зависит не только от его электрической проводимости. Было показано также, что полоски миометрия имеют неодинаковую сократительную активность при их механическом раздражении. Синхронизацию маточных сокращений обеспе- 
чивает механорецепторный механизм обратной связи, что было установлено в оригинальном опыте с коромыслом, к концам которого прикреплялись полоски миометрия. Центр коромысла крепился к дну камеры. Коромысло, как диэлектрик, не проводило электрический сигнал, исключало морфологическую связь между полосками миометрия, создавая лишь жесткую прямую механическую связь. В ответ на механические стимулы полоски миометрия синхронизировали свои сокрашения. Авторы пришли к заключению, что в основе механизма синхронизации сократительной деятельности утеромиоцитов лежит миогенный фактор: активация фазной синхронной активности утеромиоцитов, механорецепторный механизм обратной связи по растяжению миоцитов, наличие прямой механической связи между оптимально растянутыми утеромиоцитами $[5,7]$.

Таким образом, экспериментальный подход in vitro позволяет изучать молекулярные и клеточные механизмы маточного сокращения на тканевом уровне. Однако, подобный подход имеет существенные недостатки, поскольку не учитывает регуляторное влияние систем целостного организма на активность рецепторов и их лигандов. Этот факт объясняет противоречивые результаты, полученные в ряде исследованиях одного и того же явления in vivo и in vitro, поэтому большинство авторов рекомендует не ограничиваться экспериментами in vitro, а проводить исследования в условиях целостного организма.

Подход, объединяющий наблюдения in vivo и in vitro в одном исследовании был предпринят с целью выявления пролонгированных эффектов лекарственных средств на сократительную активность миометрия. В исследовании J. Hajagos-Tóth [8] было установлено, что уровень эстрогенов в крови матери влияет на сократительную активность матки на протяжении беременности и во время родов. С этой целью самкам крысам в течение беременности вводили $17 \beta$-эстрадиол. Исследование полосок миометрия этих животных показало снижение сократительного ответа на раздражение их норадреналином. В исследовании удалось установить, что этот эффект осушествляется за счет влияния эстрогенов на $\alpha 2$-адренорецепторы. Авторы делают вывод, что агонисты и антагонисты обоих подтипов $\alpha 2$-адренорецепторов могут регулировать аномальную сократительную деятельность матки. Однако эффект может оказаться видоспецифичным [8].

Moдели in situ. Для изучения локализации пейсмейкера, механизмов распространения, стимуляции, торможения маточного сокращения и процессов его ауторегуляции в литературе описан экспериментальный подход in situ. Этот подход позволяет исследовать реакции и ауторегуляцию органа в искусственной среде, имитирующей среду организма. K.Geisler и соавторы [9], а также J.Künzel и соавторы [10] использовали подобную перфузионную модель матки свиньи для изучения индукции и торможения маточной активности и выявления оптимального «окна зачатия» при выполнении вспомогательных репродуктивных технологий. Авторы изучали влияние различных доз прогестерона на индуцированные простагландинами (PG1, PGE2, PGF2 $\alpha$ ) сокращения матки. С этой целью регистрировали в полости матки синхронно on line динамику температуры и центрального венозного давления. Было показано, что индуцированные простагландинами сокращения матки после введения прогестерона дозозависимо снижались. То есть прогестерон оказывает тормозное влияние на индуцированную простагландиными сократительную активность. Авторами было выявлено также, что с целью индукции маточных сокращений следует применять простогландины Е2 и F2a (PGE2, PGF2 $\alpha$ ). Авторы подчеркнули преимущество открытой системы перфузии - скорость и легкость настраивания, управления системой перфузии одним человеком, возможность работы системы без мембранного оксигенатора и инкубатора [9-11]. Однако в процессе использования открытой перфузионной модели может наблюдается гипотермия матки, на фоне которой нарушается работа трансмембранных ионных насосов [12].

Для решения аналогичных задач W. J. Lammers и соавторы [13] предложили другой вариант перфузионной модели. Подобное исследование авторы проводили на изолированном роге беременной матки морской свинки и крысы, заполняя полость матки после её опорожнения от плодов эквивалентным объемом раствора Рингера-Тироде. Регистрацию маточной активности перфузируемой матки осуществляли с помощью цифровой видеокамеры (морские свинки) или проводили её синхронную униполярную регистрацию (у крыс). На этой модели удалось установить, что единая специфическая область пейсмейкера в матке морской свинки и крысы отсутствует, а подавляющее большинство спонтанных сокращений имеет локальный характер [13].

Известно что, пейсмейкер или водитель ритма это самопроизвольно активирующаяся клетка, генерирующая медленные волны или потенциалы действия без внешней стимуляции. До сих пор нет данных о точной локализации пейсмейкера в матке, и этот вопрос остается дискуссионным. Современные данные указывают на то, что каждое сокращение матки инициирует- 
ся в разных точках области повышенной электрической активности, т.н. «маточно-плацентарной зоне пейсмейкерной активности» $[14,15]$. На базе модели W.J. Lammers и соавторов было выполнено исследование [14], в котором проводилась многоканальная регистрация электрической активности миометрия крысы, как правило, возникающей либо в зонах плацентации в проекции края брыжейки, либо яичника [14]. В недавних исследованиях тех же авторов [16] было отмечено, что похожие пейсмейкерные зоны существуют в миометрии человека на глубине прорастания трофобласта в stratum supra vasculare [16]. В отличие от водителя ритма синоатриальном узла сердца, имеющего точную локализацию и единую структуру с постоянной периодичностью потенциалов действия, или клеток Кахаля кишечника, создающих локальные сокращения, не имеющие четкой периодичности, водитель ритма матки квазипериодичен, не имеет четкой локализации и вызывает сокращение целого органа механотрансдукционным путем $[14,15]$.

Таким образом, экспериментальная модель in situ позволила изучить характер и пути распространения сокращения миометрия, индукцию или торможение маточной активности на органном уровне, однако она не позволяет учесть комплексного влияния материнских и плодовых факторов на сократительную активность матки.

Moдели in vivo. Для устранения недостатков экспериментальных моделей in vitro и in situ, с целью изучения комбинированного влияния материнских и плодовых факторов на сократительную активность матки были использованы экспериментальные модели in vivo. Эти исследования позволяют в условиях монотерапии изучать влияние различных веществ на маточно-плацентарный кровоток и сократительную активность матки в остром и хроническом опыте в условиях живого организма. В условиях острого опыта [17] было изучено влияние терапевтических доз транквилизаторов на индуцированную сократительную деятельность у крыс в конце беременности. С этой целью авторы наркотизировали крыс в конце беременности и выполняли лапаротомию. Для регистрации электромиографической активности в дистальный отдел матки авторы вживляли электроды. Регистрацию осуществляли с помощью восьмиканального электроэнцефалографа «Galileo». Препараты вводили в брюшную полость. Было показано, что транквилизаторы (реланиум, феназепам, грандаксин), введенные на фоне индуцированной окситоцином (0,5 MЕ) сократительной деятельности, снижают амплитуду и частоту сокращений миометрия [17]. Анализируя условия проведения острых опытов in vivo, следует отметить, что при исследовании наркотизированное животное находится в неестественном положении, вследствие чего происходит нарушение маточноплацентарной гемодинамики. Подобные недостатки делают эту модель неприемлемой при планировании дизайна исследования у беременных животных, особенно при исследовании функционального состояния плода. Изучение сократительной активности матки, неразрывно связанной с функциональным состоянием плода, предпочтительнее проводить в условиях, максимально приближенных к физиологическим: естественное положение животного и отсутствие наркоза. Хронический опыт дает возможность длительной сопряженной регистрации маточной активности и сердечного ритма самки и плодов на различных этапах беременности и во время родов.

В исследованиях на беременных крысах проводилось сопоставление эффективности регистрации маточных сокращений с передней брюшной стенки животного и непосредственно с поверхности матки $[18,19]$. С этой целью на 18-й день беременности самкам интраоперационно вводили электроды в дистальный отдел матки для регистрации электромиографической активности и внутриполостной датчик для регистрации внутриматочного давления. В дальнейшем до конца беременности у ежедневно наркотизированных животных проводили синхронную регистрацию электрической активности матки с передней брюшной стенки и непосредственно с поверхности матки, а также определяли внутриматочное давление. Было установлено, что электрическая активность с поверхности брюшной стенки регистрировалась только непосредственно перед родами. Наибольшей чувствительностью в отношении паттернов маточных сокращений обладала регистрация с поверхности матки. Значимые изменения внутриматочного давления вместе с высокоамплитудными маточными сокращениями были зарегистрированы с передней брюшной стенки во время срочных и преждевременных родов, вызванных прогестином. По мнению авторов, комбинированная оценка электромиографической активности может быть использована для изучения патогенеза физиологических и преждевременных родов, а также влияния утеротонических и утеролитических средств [18, 19]. Однако результаты, в этом исследовании были получены у наркотизированных животных, находящихся в неестественном положении, при отсутствии мониторинга функционального состояния плодов. Последний чрезвычайно важен при планировании дизайна исследования, поскольку его назначение должно улучшать или, по крайней мере, не ухудшать функционального состояния плодов из-за наркоза и длительного неестественного положения животного. 
DOI: 10.25557/0031-2991.2020.01.155-161

Длительная синхронная регистрация сердечного ритма и сократительной активности матки у беременных самок и плодов кролика проводилась в хроническом опыте на различных этапах беременности и родах [20]. На 28-ой день беременности авторы вживляли электроды в дистальный отдел матки для регистрации электрической и механической активности миометрия, в бурый жир - для регистрации сердечного ритма плодов и в холку самки - для регистрации её ЭКГ. Через два дня у денаркотизированного животного, находящегося в естественном положении, проводили регистрацию электромиографической активности, ЭКГ самки и плодов, а также определяли внутриматочное давление на электроэнцефалографе «Биоскрипт». Было показано, что инфузия фосфокреатина при родах индуцированных окситоцином регулирует маточную активность, а двукратное увеличение дозы препарата приводит к гипертонусу матки. О характере родовой деятельности авторы судили также по изменению сердечного ритма плодов [20]. Однако, результаты полученные в хроническом опыте, могут иметь видоспецифичные особенности. Авторы считают, что запуск процесса родов у человека и животных могут различаться и эти особенности надо учитывать в экстраполяции результатов исследований на человека [21]. Например, известно, что роды у овец и коров инициируются фетальными гипоталамо-гипофизарными механизмами, обуславливающими прогрессивное уменьшение соотношения прогестерона и эстрогена. Однако у человека таких значительных изменений синтеза стероидов не происходит [22].

Таким образом, исследования in vivo предпочтительнее осуществлять в хроническом опыте, что позволяет адекватно анализировать патогенез и нарушения сократительной деятельности матки и функционального состояния плода с учетом регуляторного влияния нервной и эндокринной систем организма матери, а также позволяет оценивать эффект монотерапии утеротоническими и утеролитическими препаратами. При этом экспериментальные должны быть подтвержденв в клинических исследованиях.

Моделирование in silico. В последние годы в связи с развитием компьютерных технологий появилась возможность создания объемной реконструкции органов, в том числе матки, и разработки новейших алгоритмов для прогноза развития аномалий родовой деятельности. При помощи компьютерных технологий на основе данных, полученных в экспериментах на изолированных полосках миометрия и при выполнении электрогистрографии (ЭГГ) у женщин, проводится математическое моделирование in silico. Данный экспериментальный подход используют при изучении фундаментальных свойств сократительной активности миометрия человека. Такое моделирование - важный вклад в неинвазивное изучение электрической проводимости миометрия и разработку новых технологий мониторинга сократительной активности матки. В условиях модели можно анализировать реакцию системы на различные стимулы и выявлять нарушения расчетных физиологических закономерностей. Данный подход позволяет оценивать распространение гладкомышечного возбуждения в матке в конце беременности у человека [22, 23].

В исследованиях S. Rihana и C. Marque [24], а также L. Bursztyn и соавторов [25] удалось максимально корректно смоделировать возникновение клеточного потенциала действия в миометрии. Авторы сформулировали алгоритм, описывающий связь между возбуждением и сокращением, как функцию большого количества электрофизиологических параметров, связанных с изменениями концентраций ионов потенциала действия (ПД) $[24,25]$. На основе данных, полученных с помощью наружной ЭГГ, была разработана аналитическая модель паттерна электрической активности миометрия [26]. ЭГГ матки регистрировалась в конце беременности у женщин сетью из 64 электродов высокой плотности, расстояние от миометрия до передней брюшной стенки измерялось с помощью электрографии. Модель описывает электрический сигнал на клеточном уровне, его распространение в миометрии и проводимость к поверхности брюшины. Модель точно описывает проводимость сигнала от миометрия к коже с помощью ограниченного числа параметров [26]. На основе этих данных были созданы различные многоуровневые модели электрической активности миометрия у женщин. Так, модель [26] была дополнена данными, полученными другими авторами в конце беременности с помощью магнитомиографии (ММГ) на сверхчувствительных магнитомометрах SQUID. P.S.La Rosa и соавторы [27] на основе данных ЭГГ и ММГ построили упрощенную геометрическую модель сократительной активности матки у женщин в конце беременности. В этой многоуровневой модели матка представлена в виде сферы с одним водителем ритма, локализованным в области дна [27]. Эта модель была дополнена в новом исследовании M. Zhang и соавторов [22]. Авторы разработали математическую модель, в которой для моделирования геометрии объема матки учитывается не только чувствительность ММГ, но и ориентация функциональных модулей матки, а также расположение пейсмекера. По мнению авторов, данную модель можно использовать как неинвазивный метод оценки паттерна сокращения матки, расширяющий клинические представления о сократительной активности матки и анома- 


\section{DOI: 10.25557/0031-2991.2020.01.155-161}

лиях родовых сил [22]. Следовательно, моделирование in silico расширяет возможности исследований у человека интранатальной маточной активности, патогенеза аномалий родовых сил и путей их медикаментозной коррекции. Однако моделирование in silico основано на упрощенной анатомии матки, строится только на данных электрофизиологии и не учитывает особенности метаболизма утеромиоциотов. С этим утверждением согласны и другие авторы, доказавшие, что для создания комплексных моделей срочных и преждевременных родов необходимо интегрировать электрофизиологические и молекулярные данные [28].

Таким образом, сопоставление преимуществ и недостатков моделей in vitro, in situ, in vivo, in silico и их сочетаний позволяет осуществить выбор экспериментального подхода для решения проблем физиологического и патологического акушерства согласно поставленным задачам. При этом хронический опыт на животных, имеющих гемохориальный тип плаценты, позволяет проводить синхронную регистрацию электрической активности матки и сердечного ритма плодов с последующими биохимическими и морфологическими исследованиями плодово-плацентарного и материнского материала. Подобный, максимально приближенный к клиническим условиям дизайн, целесообразно использовать для изучения влияния лекарственных средств, обладающих аналгетическими, утеролитическими, утеротоническими, антиоксидантными, антигипоксантными свойствами в монотерапии. Кроме того модель можно считать адекватной для проведения фундаментальных исследований по изучению патогенеза аномалий сократительной деятельности матки и путей их коррекции.

\section{Литература}

1. Константинова Н.Н., Павлова Н.Г. Развитие представлений об универсальных гемодинамических реакциях в функциональной системе мать-плацента-плод. Журнал акушерства и женских болезней. 2004; 53(1): 27-30.

2. Савицкий Г.А., Савицкий А.Г. Биомеханика физиологической и патологической родовой схватки. СПб; «Элби», 2003.

3. Железова М.Е. Быстрые роды в современном акушерстве. Практическая медицина. 2016; 1(93): 16-21.

4. Ившин А.А., Гуменюк Е.Г., Шифман Е.М. Колыбина П.В., Гарлоева Ю.Н. Влияние родостимуляции на церебральную гемодинамику плода. Журнал акушерства и женских болезней. 2013; 62(2): 93-100.

5. Савицкий А.Г., Савицкий Г.А., Милль К.В. Фундаментальные механические свойства миометрия человека. СПб; «Элби-Спб», 2013.

6. Balki M., Ramachandran N., Lee S., Talati C. The Recovery Time of Myometrial Responsiveness After Oxytocin-Induced Desensitization in Human Myometrium In Vitro. Anesthesia \& Analgesia. 2016; 122(5): 1508-15.

7. Савицкий А.Г., Савицкий Г.А., Иванов Д.О., Милль К.В. О миогенном механизме синхронизации и координации сокраще- ний утеромиоцитов во время родовой схватки человека. Бюллетень Федерального центра сердца, крови и эндокринологии им. В.А. Алмазова. 2012; 3(14): 33-40.

8. Hajagos-Tóth J., J. Bóta, Ducza E., Csányi A., Tiszai Z., Borsodi A., et al The effects of estrogen on the $\alpha 2$-adrenergic receptor subtypes in rat uterine function in late pregnancy in vitro. Croat Med J. 2016; 57(2): 100-9.

9. Geisler K., Künzel J., Grundtner P., Müller A., Beckmann M. W., Dittrich R. The perfused swine uterus model: long-term perfusion. Reproductive Biology and Endocrinology, 10:110: 1-10. 2012.

10. Künzel J., Geisler K., Maltaris T., Müller A., Hoffmann I., Schneider H., et al Effects of Interactions Between Progesterone and Prostaglandin on Uterine Contractility in a Perfused Swine Uterus Model. In Vivo. 2014; 28(4): 467-75.

11. Самоделкин Е.И., Меркучева Н.Г., Косарева П.В., Нестерова Л.Ю. Роль простагландинов и циклооксигеназы в патогенезе хронического эндометрита. Патологическая физиология и экспериментальная терапия. 2017; 61(2): 98-100.

12. Bulletti C.S., de Ziegler $D$. Uterine contractility and embryo implantation. Curr Opin Obstet Gynecol. 2005; 17: 265-76.

13. Lammers W. J. E. P., Stephen B., Al-Sultan M. A., Subramanya S. B., Blanks A. M. The location of pacemakers in the uteri of pregnant guinea pigs and rats. Am J Physiol Regul Integr Comp Physiol. 2015; 309: R1439-R1446.

14. Young R.C. The uterine pacemaker of labor. Best Practice \& Research Clinical Obstetrics \& Gynaecology. 2018; 52: 68-87.

15. Lutton E.J., Lammers W.J.E.P., James S., van den Berg H.A., Blanks A.M. Identification of uterine pacemaker regions at the myometrial-placental interface in the rat. J Physiol. 2018; 596(14): 2841-52.

16. Lutton E.J., Lammers W.J., James S., van den Berg H.A., Blanks A.M. A computational method for three-dimensional reconstruction of the microarchitecture of myometrial smooth muscle from histological sections. PLoS One. 2017; 16; 12(3): e0173404.

17. Абрамченко В.В., Убайдатова Б.А., Корхов В.В., Петросян М.А. Влияние транквилизаторов (грандаксин, феназепам, реланиум) на сократительную активность матки беременных животных. Журнал акушерства и женских болезней. 2005; 54(3): 70-3.

18. Buhimschi C.S., Saade G.R., Buhimschi I.A., Gokdeniz R., Boyle M.B., Garfield R.E. Effect of stimulatory and inhibitory drugs on uterine electrical activity measured noninvasively from the abdominal surface of pregnant rats. Am J Obstet Gynecol. 2000; 183: 68-75.

19. Buhimschi C., Garfield R.E. Uterine contractility as assessed by abdominal surface recording of electromyographic activity in rats during pregnancy. Am J Obstet Gynecol. 1996; 174(2): 744-53.

20. Назарова Л.А., Константинова Н.Н., Толибова Г.Х., Базиан Е.В., Морозов Т.Е., Павлова Н.Г. Сократительная активность матки самок кролика в конце беременности и метод ее регистрации. Бюллетень экспериментальной биологии и медицины. 2007; 144(9):355-58.

21. Mitchell B. F., Taggart M. J. Are animal models relevant to key aspects of human parturition? Am J Physiol Regul Integr Comp Physiol. 2009; 297: R525-R545.

22. Zhang M., Tidwell V., La Rosa P. S., Wilson J. D., Eswaran H., Nehorai A. Magnetomyograms of Uterine Contractions during Pregnancy Using a Multiscale Forward Electromagnetic Approach. PLOS ONE. 2016; 11(3): $0152421-23$.

23. Sharp G.C., Saunders P.T.K., and Norman J.E. Computer models to study uterine activation at labour. Mol. Hum. Reprod. 2013; 19(11): 711-7.

24. Rihana S., Marque C. Preterm labor-modeling the uterine electrical activity from cellular level to surface recording. Conf Proc IEEE Eng Med Biol Soc. 2008; 3726-9. 
25. Bursztyn L., Eytan O., Jaffa A.J., Elad D. Mathematical model of excitation-contraction in a uterine smooth muscle cell. Amer. J. Physiol.Cell. Physiol. 2007; 292: C1816-C1829.

26. Rabotti C., Mischi M., Beulen L., Oei G., Bergmans J.W.M. Modeling and Identification of the Electrohysterographic Volume Conductor by High-Density Electrodes. IEEE Translations on Biomedical Engineering. 2010; 57(3): 519-27.

27. La Rosa P.S., Eswaran H., Preissl H., Nehorai A. Multiscale forward electromagnetic model of uterine contractions during pregnancy. BMC Med Phys. 2012; 12: 4.

28. Aslanidi O., Atia J., Benson A.P., H.A. van den Berg, Blanks A.M., Choi C., et al. Towards a computational reconstruction of the electrodynamics of premature and full term human labour. Prog Biophys Mol Biol. 2011; 107: 183-92.

\section{References}

1. Konstantinova N.N., Pavlova N.G. Concept development of complex hemodynamic reactions to the mother-placenta-fetus functional system. Zhurnal akusherstva i zhenskikh bolezney. 2004; 53(1): $27-$ 30. (in Russian)

2. Savitskiy G.A., Savitskiy A.G. Biomechanics of physiological and pathological contraction. [Biomekhanika fiziologicheskoy i patologicheskoy rodovoy skhvatki]. Sankt-Peterburg; «Jelbi», 2003. (in Russian)

3. Zhelezova M.E. Metrypercinesia in modern obstetrics. Prakticheskaya meditsina. 2016; 1(93): 16-21. (in Russian)

4. Ivshin A.A., Gumenjuk E.G., Shifman E.M. Kolybina P.V., Garloeva Ju.N. Labor induction effect on fetal cerebral hemodynamics. Zhurnal akusherstva i zhenskikh bolezney. 2013; 62(2): 93-100. (in Russian)

5. Savitskiy A.G., Savitskiy G.A., Mill' K.V. Fundamental mechanical properties of human myometrium. [Fundamental'nye mekhanicheskie svoystva miometriya cheloveka]. Sankt-Peterburg; «Jelbi- SPb», 2013. (in Russian)

6. Balki M., Ramachandran N.; Lee S.; Talati C. The Recovery Time of Myometrial Responsiveness After Oxytocin-Induced Desensitization in Human Myometrium In Vitro. Anesthesia \& Analgesia. 2016; 122(5): 1508-15.

7. Savickij A.G., Savickij G.A., Ivanov D.O., Mill' K.V. About the muscular mechanism of synchronization and coordination of uterine contractions of during the human delivery. Bulleten' Federal'nogo tsentra serdtsa, krovi i endokrinologii im. V.A. Almazova. 2012; 3(14): 33-40. (in Russian)

8. Hajagos-Tóth J., Bóta J., Ducza E., Csányi A., Tiszai Z., Borsodi A., et al The effects of estrogen on the $\alpha 2$-adrenergic receptor subtypes in rat uterine function in late pregnancy in vitro. Croat Med J. 2016; 57(2): 100-9

9. Geisler K., Künzel J., Grundtner P., Müller A., Beckmann M. W., Dittrich R. The perfused swine uterus model: long-term perfusion. Reproductive Biology and Endocrinology. 2012; 10:110: 1-10.

10. Künzel J., Geisler K., Maltaris T., Müller A., Hoffmann I., Schneider H., et al Effects of Interactions Between Progesterone and Prostaglandin on Uterine Contractility in a Perfused Swine Uterus Model. In Vivo. 2014; 28(4): 467-75.

11. Samodelkin E.I., Mercucheva N.G., Kosareva P.V., Nesterova L.Y.The role of prostaglandins and Cyclooxygenase in pathogenesis of chronic endometritis. Patologicheskaya Fiziologiya i Eksperimental'naya terapiya. 2017; 61(2): 98-100. (in Russian)
12. Bulletti C.S., de Ziegler D. Uterine contractility and embryo implantation. Curr Opin Obstet Gynecol. 2005; 17: 265-76.

13. Lammers W.J.E.P., Stephen B., Al-Sultan M.A., Subramanya S.B., Blanks A.M. The location of pacemakers in the uteri of pregnant guinea pigs and rats. Am J Physiol Regul Integr Comp Physiol. 2015; 309 : $\mathrm{R} 1439-\mathrm{R} 1446$

14. Young R.C. The uterine pacemaker of labor. Best Practice \& Research Clinical Obstetrics \& Gynaecology. 2018; 52: 68-87.

15. Lutton E.J., Lammers W.J.E.P., James S., van den Berg H.A., Blanks A.M. Identification of uterine pacemaker regions at the myometrial-placental interface in the rat. $J$ Physiol. 2018; 596(14): 2841-2852.

16. Lutton E.J., Lammers W.J., James S., van den Berg H.A., Blanks A.M. A computational method for three-dimensional reconstruction of the microarchitecture of myometrial smooth muscle from histological sections. PLoS One. 2017; 16; 12(3):e0173404.

17. Abramchenko V.V., Ubaydatova B.A., Korhov V.V., Petrosjan M.A. Effect tranquilizers (grandaxinum, phenazepam, relanium) uterine contractile activity in pregnant animals. Zhurnal akusherstva $i$ zhenskikh bolezney. 54(3): 70-73.2005; (in Russian)

18. Buhimschi C.S., Saade G.R., Buhimschi I.A., Gokdeniz R., Boyle M.B., Garfield R.E. Effect of stimulatory and inhibitory drugs on uterine electrical activity measured noninvasively from the abdominal surface of pregnant rats. Am J Obstet Gynecol. 2000; 183: 68-75.

19. Buhimschi C., Garfield R.E. Uterine contractility as assessed by abdominal surface recording of electromyographic activity in rats during pregnancy. Am J Obstet Gynecol. 1996; 174(2): 744-53.

20. Nazarova L.A., Konstantinova N.N., Tolibova G.H., Bazian E.V., Morozov T.E., Pavlova N.G. Rabbit uterine contractile activity in late pregnancy and the method of its registration. Bjulleten' jeksperimental'noj biologii i mediciny. 2007; 144 (9): 355-8. (in Russian)

21. Mitchell B. F., Taggart M. J. Are animal models relevant to key aspects of human parturition? Am J Physiol Regul Integr Comp Physiol. 2009; 297: R525-R545.

22. Zhang M., Tidwell V., La Rosa P. S., Wilson J. D., Eswaran H., Nehorai A. Magnetomyograms of Uterine Contractions during Pregnancy Using a Multiscale Forward Electromagnetic Approach. PLOS ONE. 2016; 11(3):e0152421-23.

23. Sharp G.C., Saunders P.T.K., and Norman J.E. Computer models to study uterine activation at labour. Mol. Hum. Reprod. 2013; 19(11): 711-7.

24. Rihana S., Marque C. Preterm labor-modeling the uterine electrical activity from cellular level to surface recording. Conf Proc IEEE Eng Med Biol Soc. 2008; 3726-9.

25. Bursztyn L., Eytan O., Jaffa A.J., Elad D. Mathematical model of excitation-contraction in a uterine smooth muscle cell. Amer. J. Physiol.Cell. Physiol. 2007; 292: C1816-C1829.

26. Rabotti C., Mischi M., Beulen L., Oei G., Bergmans J.W.M. Modeling and Identification of the Electrohysterographic Volume Conductor by High-Density Electrodes. IEEE Translations on Biomedical Engineering. 2010; 57(3): 519 - 27.

27. La Rosa P.S., Eswaran H., Preissl H., Nehorai A. Multiscale forward electromagnetic model of uterine contractions during pregnancy. BMC Med Phys. 2012; 12: 4.

28. Aslanidi O., Atia J., Benson A.P., H.A. van den Berg, Blanks A.M., Choi C., et al. Towards a computational reconstruction of the electrodynamics of premature and full term human labour. Prog Biophys Mol Biol. 2011; 107: 183-92.

\section{Сведения об авторах:}

Яковлева Анастасия Александровна, науч. сотр. лаб. патофизиологии ФГБОУ ВО ПСПбГМУ им. И.П. Павлова Минздрава России, e-mail: barabulka875@gmail.com;

Павлова Наталия Григорьевна, доктор мед. наук, проф., каф. акушерства и гинекологии ФГБОУ ВО ПСПбГМУ им. И.П. Павлова Минздрава России, e-mail: ngp05@yandex.ru 